\title{
Evidence based psychotherapy: special case or special pleading?
}

Paradoxically, the more firmly psychological therapies are established as bona fide interventions within mainstream mental health services, the greater the case for evidence based practice. Patients, often in severe distress, make a major personal commitment to these therapies and have a right to know that they are safe and effective. Although psychological therapies do benefit patients, ${ }^{1}$ there is also evidence that patients' mental health can deteriorate if therapies are inappropriate or carried out incompetently. ${ }^{2}$ Finally, psychological therapy represents a substantial economic investment and it could be seen as unethical to waste this money on ineffective treatments.

Evidence based psychotherapy has therefore been promoted internationally, albeit with different emphases in different health care systems. For example, the American trend, fuelled by third party payment systems in managed care, has been to compile lists of "empirically supported treatments". ${ }^{4}$ This provoked disquiet among researchers and practitioners alike. ${ }^{5}$ The National Health Service (NHS) in England has so far resisted this approach, arguing for a broader model of evidence based practice that includes both efficacy and effectiveness evidence, acknowledges the role of factors common to all therapies, and emphasises quality of service delivery. ${ }^{6}$

Systematic collation and appraisal of findings is indispensable for informed practice, commissioning or policy making. Quantitative summaries of treatment effects are not new in this field, which indeed was one of the first to apply meta-analytic techniques. ${ }^{7}$ Mackay and Barkham ${ }^{8}$ appraised systematic reviews of psychological therapies for common mental health problems in adults, against Oxman and Guyatt's 8 quality criteria. They found 42 reviews meeting all 8, and a further 43 meeting 6 or 7 . Cochrane reviews of psychological therapies are becoming available, for example on treatment of deliberate self harm, ${ }^{10}$ debriefing in adults exposed to trauma ${ }^{11}$ and chronic fatigue. ${ }^{12}$ Others, on psychological treatments for eating disorders, ${ }^{13}$ depression $^{14}$ and counselling in primary care ${ }^{15}$ are in preparation. As more systematic reviews are completed, gaps in evidence and methodological shortcomings are continually identified, and pressure builds for good research to be commissioned to address them. There are other indications that evidence based psychotherapy is an idea whose time has come. Special issues of the journals Clinical Psychology: Science and Practice (1996) and Psychotherapy Research (1998) have appeared on the topic, and of course, Evidence-Based Mental Health has treated psychological and pharmacological interventions identically in its search for best evidence of what works in the mental health field. The first evidence based guideline on treatment choice decisions in psychological therapies is soon to be published. ${ }^{16}$

So, is all for the best in the best of all possible worlds? Not entirely-there remains considerable debate about and distrust of the principles of applying research evidence to change psychotherapy practice. Some practitioners are profoundly sceptical, and fear that far from improving care, the enterprise potentially does harm. ${ }^{17}{ }^{18}$ Scepticism about the wisdom of changing practice on the basis of research evidence is fuelled by the methodological problems of psychotherapy research. This leads to fears that unreliable or even misleading evidence may be prematurely and simplistically applied. More radically, some believe that psychotherapy, by its very nature, is inimical to the research paradigms of evidence based medicine.

Some problems with trials research are remediable. For example, psychological therapy is more difficult to deliver to a set standard compared with a drug treatment. Some negative results may result from poor quality delivery of the intervention and adherence to a treatment manual does not overcome this problem. ${ }^{19}$ The very act of randomisation may produce systematic between-group differences when patients have strong preferences for one therapy over another. ${ }^{20}$ High rates of attrition ("drop out") within randomised trials sometimes create problems with interpreting the results. There are widespread practical difficulties in achieving textbook randomisation in psychotherapy trials. Despite randomisation, where samples are small there are often unplanned differences between the groups on important variables such as symptom severity. Most comparative outcome trials fail to distinguish between the effectiveness of different types of psychological therapy, for reasons that may be methodological, such as insufficient statistical power. ${ }^{21}{ }^{22}$ Many of the researchers who conduct comparative outcome trials are not in equipoise but on the contrary are enthusiasts for one therapy approach; this may influence outcome to the extent that removing the effect of allegiance may alter the result of meta-analysis. ${ }^{23}$ Individual therapists vary in effectiveness and, in most trials, such effects are unanalysed or unreported despite implications for interpreting the findings. ${ }^{24}$

Although these problems are ubiquitous, few of them are unique to psychotherapy trials and, in principle, they can all be addressed by improved research designs, including different designs to address different questions. This would mean adequately powered trials with clinically realistic samples and interventions and clinically meaningful comparison groups. ${ }^{25}$ We would see both pragmatic trials with health status utility measures and economic evaluation, and explanatory trials making planned comparisons of specific therapy "ingredients". ${ }^{26} 27$ The effect of attrition would invariably be analysed using "intention to treat" samples and the variance due to individual therapists would also be routinely reported. There would be greater awareness of the effects of patient preference, and the use of partially randomised preference trials where needed. ${ }^{28}$ Outcomes should be reported in terms of clinical as well as statistical significance. ${ }^{29}$ Equipoise of researchers could become a significant factor in decisions to fund a trial.

Some problems are more fundamental than these though, and often derive from treating psychological therapies as if they are the same as pharmacological treatments. However, the "drug metaphor" breaks down at a number of points. By its 
nature, therapist and patient cannot be blinded to the intervention being delivered, and there are profound conceptual difficulties with "placebo" treatments in psychological therapy. The intervention can never be entirely specified or standardised, as therapists are responsive to emergent issues, changing what is being delivered throughout the course of treatment. ${ }^{30}$

Problems with external validity, even in good, clinically realistic trials, are inescapable - there are intrinsic tensions between the internal validity prized by researchers and the external, ecological validity essential to clinicians. Some unavoidable threats to external validity are homogeneity of patient samples, exclusion criteria, standardisation of treatment and randomised allocation. Randomised controlled trials (RCTs) are therefore only one part of a research cycle; most appropriately they test the capabilities of a well developed therapy after single case studies and before large scale field trials. ${ }^{31}$ Both efficacy and effectiveness research is needed for evidence based practice and other research strategies complement the controlled trial. Two other sources of evidence are large sample surveys of psychotherapy recipients, and research on psychotherapeutic processes.

Practice based research is vital. We lack even elementary information about the effect of treatment setting on the outcome of therapy because such research is difficult to design, and systematic comparisons are rare. Most psychological therapy in the NHS is pragmatic and eclectic, where therapists use a judicious mix of techniques drawn from varying theoretical frameworks. Psychotherapy research, on the other hand, focuses on standardised interventions of "pure" types of therapy (eg, cognitive, behavioural, or psychoanalytic). The most prevalent interventions are paradoxically the least researched. There is sparse evidence about the most effective ways to deliver therapy or which factors influence access to therapy in the real world. The differences between effect sizes obtained in RCTs and those in everyday practice have still not been studied systematically, despite early attempts to investigate this important issue. ${ }^{32}$

Practice research networks are a promising development, where members agree to gather and pool data relating to clinical outcomes, using the same set of measures, in order to enable analysis of national or regional datasets. Data are aggregated and anonymous and individual patient details are not identifiable, although patient consent to this use of data is still advisable. These networks help practitioners to take a research based approach to exploring outcomes in their own services, sharing the findings, which can be compared with results obtained in more controlled experimental conditions. ${ }^{33}$ By developing high quality clinical databases on large clinically representative samples, practice research networks can contribute an important source of evidence on the effectiveness of services as delivered. This approach is starting to bear fruit in other fields, such as surgery, obstetrics, haematology and oncology. ${ }^{34}$

Obtaining good quality, replicated research findings on all therapeutic approaches across all diagnoses in their many complex combinations, is a huge unfulfilled enterprise. Some see the attempt as misguided and not feasible. ${ }^{25}$ An alternative approach overcomes the problem of having to test every "brand name" therapy, by paying less attention to self styled differences between therapies and instead studying basic psychological processes common to all successful therapy, such as therapeutic alliance, emotional assimilation and phases of change. Returning momentarily to the drug metaphor, psychotherapy outcome research without process research is like undertaking drug efficacy trials without laboratory research on their mode of action.

There is perhaps one sense in which psychological treatments are disadvantaged compared with drug treatments. Like health services research and research on social interventions, the cost of researching psychotherapy is borne almost entirely by public funds. For psychological therapies however, "head to head" comparisons with medication are commonly made in primary research and meta-analysis. No patents are granted on psychological therapies and as they are not commercially exploitable, the research investment in them is trifling, compared with research and development budgets in pharmaceuticals.

So is evidence based psychotherapy a special case or is there too much special pleading? Perhaps predictably, both statements are true to an extent. Evidence based psychotherapy needs some modification of the rules of evidence based medicine, but these adaptations are few, and in my view, consensus could be reached on what they are. The field certainly needs more research and development investment, in under researched therapies, modalities and populations. But claims that psychotherapy, in any variant, is uniquely outside the discourse, are insupportable.

$$
\begin{array}{r}
\text { GLENYS PARRY, DipClinPsych, PhD, CPsychol, FBFsS } \\
\text { University of Sheffield School of Health and Related Research } \\
\text { Sheffield,UK } \\
\text { email: g.d.parry@sheffield.ac.uk }
\end{array}
$$

1 Roth AD, Fonagy P. What Works for Whom? A Critical Review of Psychotherapy Research. New York: Guilford Press, 1996.

2 Lambert MJ, Bergin AE. The effectiveness of psychotherapy. In: Bergin AE, Garfield SL, eds. Handbook of Psychotherapy and Behavior Change, $4^{\text {th }}$ ed. New York: John Wiley and Sons, 1994;141-50.

3 American Psychological Association Task Force on Psychological Intervention Guidelines. Template for developing guidelines. Interventions for mental disorders and psychosocial aspects of physical disorders. Washington DC: American Psychological Association, 1999

4 Chambless DL. Task force on promotion and dissemination of psychological procedures. A report adopted by the Division 12 Board, October 1993. Washington: American Psychological Association, 1993.

5 Elliott R. Editor's introduction: a guide to the empirically supported treatments controversy. Psychotherapy Research 1998;8:115-25.

6 NHS Executive. NHS psychotherapy services in England: review of strategic policy. London: Department of Health, 1996.

7 Smith ML, Glass GV. Meta-analysis of psychotherapy outcome studies. Am Psychol 1977;32:752-60.

8 Mackay HC, Barkham M. Report to the National Counselling and Psychological Therapies Clinical Guidelines Development Group: evidence from published reviews and meta-analyses, 1990-98. Psychological Therapies Research Centre, University of Leeds, 1998.

9 Oxman $\mathrm{AD}$, Guyatt GH. Guidelines for reading literature reviews. CMAJ 1988;138:697-703.

10 Hawton K, Arensman E, Townsend E, et al. Deliberate self-harm: the efficacy of psychosocial and pharmacological treatments (Cochrane review). In: The Cochrane Library, Issue 3, 1998. Oxford: Update Software.

11 Wessely S, Rose S, Bisson J. Brief psychological interventions ("debriefing") for immediate trauma related symptoms and the prevention of post traumatic stress disorder (Cochrane review). In: The Cochrane Library, Issue 4, 1999. Oxford: Update Software

12 Price JR, Couper J. Cognitive behaviour therapy for chronic fatigue syndrome in adults (Cochrane review). In: The Cochrane Library, Issue 4, 1999. Oxford: Update Software.

13 Hay PJ, Bacaltchuk J. Psychotherapy for bulimic nervosa and binging (Cochrane review). In: The Cochrane Library, Issue 4, 1999. Oxford: Update Software.

14 Hunot V, Churchill R. Systematic review of brief psychological treatments for depression (Cochrane review in preparation). Personal communication, 1999.

15 Rowland N, Bower P, Mellor-Clark J, et al. The effectiveness and cost-effectiveness of counselling in primary care (Cochrane Review in preparation).

16 Parry G. Guideline on treatment choice decisions for psychological therapies. Audit Trends 1998;6:110.

17 Taylor D. Critical review of the review of psychotherapy services in England. Psychoanalytic Psychotherapy 1998;12:111-8.

18 Henry WP. Science, politics and the politics of science: the use and misuse of empirically validated treatment research. Psychotherapy Research 1998;8:126-40.

19 Waltz J, Addis ME, Koerner K, et al. Testing the integrity of a psychotherapy protocol: assessment of adherence and competence.J Consult Clin Psychol 1993;61:620-30.

20 Bradley C. Psychological issues in clinical trial design. Irish Journal of Psychology 1997; 18:67-87.

21 Kazdin AE, Bass D. Power to detect differences between alternative treatments in comparative psychotherapy outcome research.J Consult Clin Psychol 1989;57:138-47. 
22 Norcross JC. Dispelling the dodo bird verdict and the exclusivity myth in psychotherapy. Psychotherapy 1995;32:500-4.

23 Robinson LA, Berman JS, Neimeyer RA. Psychotherapy for the treatmen of depression: a comprehensive review of controlled outcome research. Psychol Bull 1990;108:30-49.

24 Luborsky L, Crits-Christoph P, Woody GE, et al. Do therapists vary much in their success? Findings from four outcome studies. Am J Orthopsychiatry 1986;56:501-12.

25 Aveline M, Shapiro DA, Parry G, et al. Building research foundations for psychotherapy practice. In: Aveline M, Shapiro DA, eds. Research foundations for psychotherapy practice. Chichester; New York: J Wiley in association with the Mental Health Foundation, 1995

26 Schwartz D, Lellouch J. Explanatory and pragmatic attitudes in therapeutical trials. J Chronic Dis 1967;20:637-48.

27 Kraemer HC, Pruyn JP. The evaluation of different approaches to randomized clinical trials. Report on the 1987 MacArthur Foundation Network I Methodology Workshop. Arch Gen Psychiatry 1990;47:1163-9.
28 Brewin CR, Bradley C. Patient preferences and randomised clinical trials. BMJ 1989;299:313-5

29 Evans C, Margison F, Barkham M. The contribution of reliable and clinically significant change methods to evidence-based mental health [EBMH notebook]. Evidence-Based Mental Health 1998 Aug;1:70-2.

30 Stiles WB, Shapiro DA. Disabuse of the drug metaphor: psychotherapy process-outcome correlations. J Consult Clin Psychol 1994;62:942-8.

31 Salkovskis PM. Demonstrating specific effects in cognitive and behavioural therapy. In: Aveline M, Shapiro DA, eds. Research foundations for psychotherapy practice. Chichester; New York:J Wiley in association with the Mental Health Foundation, 1995;191-228.

32 Kirk JW. Behavioural treatment of obsessional-compulsive patients in routine clinical practice. Behav Res Ther 1983;21:57-62.

33 Barkham M, Evans C, Margison F, et al. The rationale for developing and implementing core batteries in service settings and psychotherapy outcome research. Journal of Mental Health 1998;7:35-47.

34 Black N. Developing high quality clinical databases. BMJ 1997;315:381-2.

\section{Welcome to our new associate editors}

We have two new associate editors joining our editorial team. The first, David Haslam, MD, MSc, was trained in medicine at McMaster University, Ontario, and completed his postgraduate training in psychiatry at Dalhousie University, Nova Scotia, in 1999 (picking up the Laughlin Prize of the American Psychiatric Association on the way!). He is currently a staff psychiatrist at North Bay Psychiatric Hospital in Ontario. David has extensive knowledge of psychiatry, clinical epidemiology and evidence based practice. As well as using evidence based strategies daily in his clinical practice, he has developed clinical practice guidelines (The Canadian Anxiety and Mood Disorder Network Guidelines on the treatment of bipolar disorder) and has extensive experience teaching evidence based practice, both in North America and the UK. He will particularly strengthen EvidenceBased Mental Health in its coverage of general adult psychiatry, joining the editorial team in the challenging task of extracting the most clinically useful information from the large numbers of phase III pharmaceutical trials that are published each year. We are delighted to welcome David to the editorial team and consider ourselves extremely fortunate to have secured the services of such an enthusiastic and gifted clinician.

Carolyn Bryne is another addition to our editorial team and will specifically help us with the selection of qualitative studies for Evidence-Based Mental Health. Carolyn is a professor in the School of Nursing, Faculty of Health Sciences, McMaster University, and has published extensively in the mental health field for many years. She has a particular interest in qualitative studies, their methodological rigour, and their application to psychiatric nursing. The editors are committed to making available the best and most clinically relevant qualitative studies that complement and enhance the quantitative evidence with which we are more familiar. We also are very keen to include colleagues in psychiatric nursing among our readers and commentators. We are delighted that Carolyn has accepted the challenge of helping us to reach both objectives, and we look forward to her contributions to the journal.

THE EDITORS Evidence-Based Mental Health

\section{A new addition to this journal}

There has been an explosion in the creation of evidence resources over the past several years and it is now becoming a challenge for clinicians to find the most valid and useful evidence based resources with which to practice. Beginning with this issue a section entitled "Resource Corner," will publish reviews of evidence based health care resources written by front line clinicians. These resources will include journals (primary and secondary), evidence based textbooks, computer software, and websites. In the future, each issue of the journal may contain up to 2 reviews of such products.

Only clinicians without competing interests in the resource under review will be asked to write a review. Our reviewers will be asked to consider specific methodologic guides (available to view on http://hiru.mcmaster.ca) when assessing these resource products. In particular, they will consider if the authors of the resources have identified explicit criteria for determining the validity of the evidence and if they adhere to these criteria. The reviewers will also provide a "bottom line" recommendation indicating if and how the resource could be used in practice

These resource reviews will also appear in one or both of $A C P$ Journal Club and Evidence-Based Medicine and were inspired by their editors.

THE EDITORS

Evidence-Based Mental Health 